\title{
ECR 2011: Personalisierte Medizin als Ziel
}

\author{
Bildgebende Verfahren liefern Grundlagen und frühe Informationen
}

Weil der Anteil der alten Menschen weltweit kontinuierlich steigt und auch weil Übergewicht mit seinen massiven gesundheitlichen Folgen ein immer größeres Problem wird, werden die Ausgaben des Gesundheitssystems dramatisch zunehmen. Für die USA wird beispielsweise für das Jahr 2025 ein Anteil der Gesundheitskosten am Bruttonationalprodukt von 25 Prozent prognostiziert, denn gleichzeitig steigen die Möglichkeiten der Medizin - was häufig recht kostenintensiv ist. Das Gebot der Stunde lautet daher: Gezielter Einsatz der Mittel. Die Radiologie mit ihren vielfältigen bildgebenden Verfahren bietet dafür sowohl in der Ursachenforschung, in der Diagnose, und Therapie als auch in der Therapiekontrolle gezielt einsetzbare Werkzeuge. Neueste

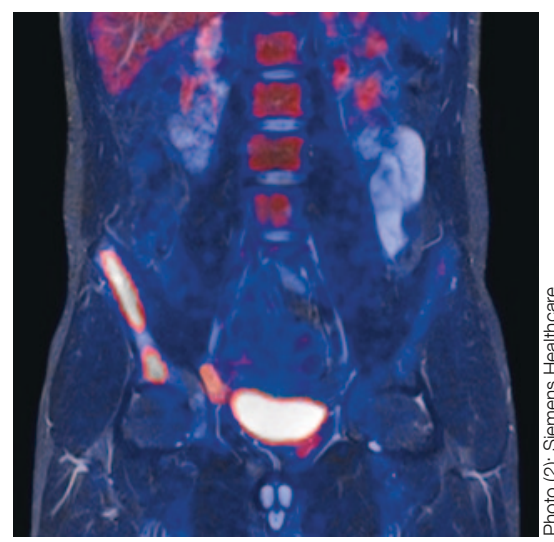

mMR-Darstellung eines Tumorgeschehens am Knochenmark.

Entwicklungen ebenso wie die Grundlagen als unerlässliches Fundament standen Anfang März in Wien beim Europäischen Radiologenkongress (ECR) zur Diskussion. Mit 19.000 Teilnehmern ist der ECR das weltweit zweitgrößte Ereignis des Fachs - übertroffen lediglich vom Nordamerikanischen Pendant (RSNA).

\section{Mehr Informationen erfordern mehr Wissen}

Personalisierte Medizin ist der Schlüssel zu einem besseren Resultat, stellte Prof. Maximilian F. Reiser, Dekan der Medizinischen Fakultät der Universität München und Präsident der Europäischen Radiologen Ge- sellschaft (ERS) fest: „Die richtige Gesamtbehandlung für den richtigen Patienten zur richtigen Zeit." Ein klassisches Beispiel ist, die Wahl der Bildgebungsverfahren an das individuelle Patientenrisiko anzupassen, etwa bei der Brustkrebsvorsorge. Besteht kein oder ein geringes Risiko kommt die Mammographie zum Einsatz, bei mittlerem Risiko erfolgen Mammographie und Ultra-

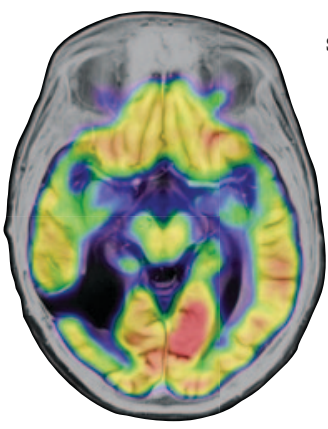

Darstellung eines Gehirntumors mit dem moklekularen MR-Ganzkörpersystem. sind, bringt die Detektion mittels molekularer Bildgebung einen Zeitvorteil. Eine neue Anwendung ist ein optisches Bildgebungsgerät zur Früherkennung von Arthritis. Und auch der Ultraschall für die molekulare Bildgebung steht, so Kiessling, vor der klinischen Anwendung. Während also die molekulare Bildgebung bereits klinische Realität ist, befindet sich der Einsatz des Ultraschall oder Magnetresonanzuntersuchung bei Hochrisiko Mammographie und MRI. Biomarker ermöglichen heute vermehrt sowohl das Ansprechen auf als auch Resistenzen gegenüber Therapien festzustellen. Auch hier stellt die Bildgebung eine sensible Erkennungsmethode dar und kann so unterscheiden, ob ein Patient mit Aussicht auf Wirkung behandelt werden kann oder sowohl Kosten als auch Nebenwirkungen einer unwirksamen Therapie vermieden werden können. Die Fülle an Informationen, die durch die unterschiedlichen Darstellungsebenen eröffnet wird, erfordert vom Radiologen das entsprechend breite Wissen, um die Informationen auswerten zu können. „Der Radiologe ist nicht mehr nur auf Morphologie und Anatomie konzentriert, sondern“, so Reiser, „auch auf Stoffwechselvorgänge durch die funktionelle Computertomographie (CT) und Positronenemissionstomographie (PET).“ Schließlich erlauben bildgeführte Therapien ein schonenderes Vorgehen und sind, beispielsweise im Fall der Radiofrequenzablation der Leber, gleich wirksam wie die offene Chirurgie.

\section{Molekulare Bildgebung als klinische Realität}

In der Darstellung von Biomarkern spielt die molekulare Bildgebung bereits jetzt eine wichtige Rolle und wird in Zukunft noch häufiger zum Einsatz kommen, erklärte Prof. Fabian Kiessling, Vorstand des Instituts für Experimentelle Molekulare Bildgebung der Universität Aachen: „Welchen Marker wir heranziehen, ist egal, es muss früh sein.“ Da die frühesten Zeichen einer Erkrankung im Gewebe und auf der Zellebene zu finden hochgeschwindigkeits MRI mit 7 Tesla noch im experimentellen Stadium. Eines von weltweit 38 Geräten ist auf dem Gelände des AKH in Wien in Verwendung mit dem Fokus auf verbreitete Krankheiten, berichtete Prof. Siegfried Trattnig, Leiter des Exzellenzzentrums Hochfeld MR in Wien. Eine neue Initiative soll die Möglichkeiten der Bildgebung gebündelt voranbringen. Der Vienna Imaging Cluster werde sich, so Trattnig, mit Anwendungsgebieten von Kunst bis molekularer Bildgebung beschäftigen.

\section{Detektivarbeit in Sachen Kunst}

Denn auch in der Kunst bietet die Bildgebung Informationen, die auf anderem Wege nicht oder höchst aufwendig zu erhalten wären. $\mathrm{Ob}$ es sich dabei um unter Ölfarbenschichten verborgene ältere Versionen desselben Bildes handelt, wie etwa bei Francisco de Goyas „Una mañola“ oder um die Alters- und Materialbestimmung bei antiken Gegenständen. „Jeder Radiologe mit einer gewissen Neugier und Zugang $\mathrm{zu}$ einem Multidetector CT Scanner kann seine radiologischen Fähigkeiten einsetzen, um unser technisches und kulturelles Wissen über Kunst und Antiquitäten zu erweitern, im speziellen bei Terrakotta-, Holz- und Steinstatuen", meinte Dr. Marc Ghysels aus Brüssel. „Das ist zugegebenermaßen eine untypische Anwendung der Radiologie." Die Zahl der heute auf dem Markt auftauchenden authentischen Kunstwerke nimmt ab, Kopien oder Fälschungen überschwemmen den Kunstmarkt. Daher habe auch diese Anwendung weiteres großes Entwicklungspotenzial.

Quelle: ECR 2011, 3.-7. März 2011, Wien 